Sammlung Metzler

Band 233 
Hans-Gerd Winter

\section{Jakob Michael Reinhold Lenz}

Verlag J.B. Metzler Stuttgart - Weimar 
Die Deutsche Bibliothek - CIP-Einheitsaufnahme

Winter, Hans-Gerd:

Jakob Michael Reinhold Lenz / Hans-Gerd Winter.

- 2., überarb. und aktualisierte Aufl.

- Stuttgart ; Weimar : Metzler , 2000

(Sammlung Metzler; Bd. 233)

1. Aufl. u.d.'T.: Winter, Hans-Gerd: J. M. R. Lenz

ISBN 978-3-476-12233-9

SM 233

ISBN 978-3-476-12233-9

ISBN 978-3-476-04105-0 (eBook)

DOI 10.1007/978-3-476-04105-0

ISSN 05583667

Dieses Werk einschließlich aller seiner Teile ist urheberrechtlich geschützt. Jede Verwertung außerhalb der engen Grenzen des Urheberrechtsgesetzes ist ohne Zustimmung des Verlages unzulässig und strafbar. Das gilt insbesondere für Vervielfältigungen, Übersetzungen, Mikroverfilmungen und die Einspeicherung und Verarbeitung in elektronischen Systemen.

(C) 2000 Springer-Verlag GmbH Deutschland

Ursprünglich erschienen bei J.B. Metzlersche Verlagsbuchhandlung und Carl Ernst Poeschel Verlag GmbH in Stuttgart 2000 


\section{Vorwort zur 2. Auflage}

Die zweite Auflage dieses Bandes arbeitet gegenüber der ersten von 1987 die Ergebnisse der sich inzwischen rasant entwickelnden Forschung auf. Neue Texte von Lenz sind entdeckt worden, neue Perspektiven entwickelt worden, die zum Teil auch die bekannten Texte in einem anderen Licht erschienen lassen; zudem hat sich die Einstellung des Verfassers in einigen Punkten verändert; manche Wertungen oder Behauptungen haben sich als überholt erwiesen. Deswegen mußten alle Kạpitel sehr sorgfältig überarbeitet und zum Teil erheblich ergänzt werden. Beibehalten wurde die Kapiteleinteilung. Nach einem Überblick über das Lenz-Bild in der Forschung und die Geschichte der Lenz-Manuskripte folgt sie im wesentlichen der Chronologie von Lenz' Leben und der Lenzrezeption. Deutlicher als bei anderen Autoren gehen nämlich in die Werke autobiographische Momente ein, obwohl jene in diesen nicht aufgehen. Und umgekehrt hat Lenz immer wieder sein Leben unter der Folie literarischer oder religiöser Muster interpretiert, wie zum Beispiel seine Briefe belegen.

Für die Geschichte der produktiven Rezeption bietet sich eine chronologische Darstellung an, um auch die gegenseitige Beeinflussung einzelner Aneignungen hervorheben zu können. Die Bibliographie ist aus Gründen der besseren Übersichtlichkeit vollständig ans Ende des Bandes gesetzt worden. Auch sie wurde umfassend überarbeitet und stark aktualisiert. Die Bibliographie wurde mit Unterstützung von Gabriele Thal überarbeitet. Lenz-Zitate folgen, wenn nicht anders angemerkt, der Werkausgabe von Sigrid Damm mit Angabe von Band und Seitenzahl, bei Briefen wird das Datum nachgewiesen. Hinweise auf die Sekundärliteratur im fortlaufenden Text verweisen mit Verfassernamen und Jahreszahl auf die Bibliographie. Für die wertvollen Hinweise und Anregungen, die mir während des Schreibens der 2. Auflage dieses Buches zugegangen sind, bedanke ich mich. 


\section{Inhalt}

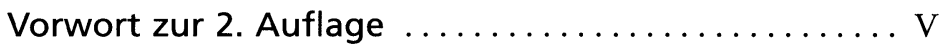

Einleitung $\ldots \ldots \ldots \ldots \ldots \ldots \ldots \ldots \ldots \ldots \ldots \ldots, 1$

1. Doch nicht nur ein "vorübergehendes Meteor «?

Die wissenschaftliche Aneignung von Lenz ...... 6

1.1 Zum Lenz-Bild der Literaturwissenschaft . . . . . . . . 6

1.2 Kurze Geschichte der Lenz-Manuskripte und Werkausgaben

2. Der »erloschene Blitz aus Livland« Lenz' Biographie und Werk .............. 26

2.1 Kindheit und Jugend $\ldots \ldots \ldots \ldots \ldots \ldots \ldots \ldots \ldots \ldots$

2.2 Studium in Königsberg. . . . . . . . . . . . . . . 29

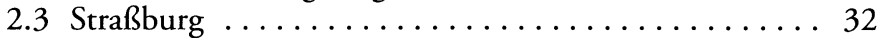

Straßburg als Herausforderung $\ldots \ldots \ldots \ldots \ldots \ldots \ldots 32$

Beschäftigung mit Theologie und Moralphilosophie ..... 42

Unglückliche Liebesbeziehungen zu Friederike Brion,

Cleophe Fibich und Cornelia Schlosser . . .......... 46

"Zerbin oder die neuere Philosophie $\ldots . . . \ldots \ldots \ldots \ldots .49$

2.4 Die Straßburger Dramen................. 52

Shakespeare- und Plautusrezeption............... 52

"Anmerkungen übers Theater $\ldots \ldots \ldots \ldots \ldots \ldots \ldots 56$

"Der Hofmeister oder die Vorteile der Privaterziehung" . . . 61

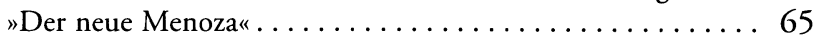

"Die Soldaten ${ }^{\prime} \ldots \ldots \ldots \ldots \ldots \ldots \ldots \ldots \ldots \ldots, 67$

"Die Freunde machen den Philosophen $« . . . \ldots \ldots \ldots . .72$

"Der Engländer" .......................... 74

Dramenfragmente .................... 76

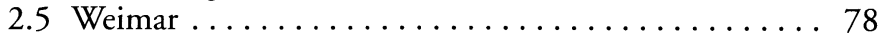

Lenz' Erfahrungen am Weimarer Hof und ihre Verarbeitung im $»$ Waldbruder" ....................... 78

Lenz' "Eseley» und der Bruch mit Goethe . . . . . . . . 83

2.6 Schweizer Reisen und Emmendingen ........... 87

Der "Landläuffer» in Emmendingen und in der Schweiz . . 87

Der Aufenthalt bei Oberlin . . . . . . . . . . . . . . . . 88 
Lenz" "Krankheit" . . . . . . . . . . . . . . . 90

"Der Landprediger" ................... 93

2.7 Riga, Petersburg und Moskau . . . . . . . . . . . 96

Lenz im russischen Reich ............... 96

In Rußland entstandene Werke . . . . . . . . . . . . 100

3. "Und mögen auch Jahrhunderte über meinen

Schädel verachtungsvoll hinwegschreiten« die produktive Rezeption Lenz' . . . . . . . . . . 104

3.1 Lenz und die zeitgenössische literarische

Öffentlichkeit ....................... 104

3.2 Erste Lenz-Aneignungen - von den Romantikern

bis Büchner . . . . . . . . . . . . . . . . . 107

Lenz in der Romantik. . . . . . . . . . . . . . . . . 107

Lenz im Elsaß . . . . . . . . . . . . . . . . 110

Büchners Auseinandersetzung mit Lenz . . . . . . . . 110

3.3 Umstrittene Kultfigur einiger Literaten -

von Büchners Tod bis zum Zweiten Weltkrieg . . . . . 117

Lenz im 19. Jahrhundert. . . . . . . . . . . . . . 117

Lenz im Umkreis von Naturalismus und Expressionismus . 119

Über Büchner vermittelte Lenz-Rezeption bei Naturalisten

und Expressionisten . . . . . . . . . . . . . . 125

Lenz bei Hofmannsthal und Kafka . . . . . . . . . . . 128

Robert Walsers "Lenz«-Fragment . . . . . . . . . . . . . 129

Lenz-Rezeption in der Weimarer Republik . . . . . . . . 130

3.4 Letzte intensive Phase der Aneignung -

von der Nachkriegszeit bis zur Gegenwart . . . . . . . 134

Brechts Lenz-Rezeption . . . . . . . . . . . . . . 135

Anknüpfungen an Lenz in der DDR-Literatur . . . . . . 140

Celan und Lenz . . . . . . . . . . . . . . . . . . . . 147

Lenz-Rezeption vor der Studentenbewegung . . . . . . . . . 149

Heinar Kipphardts Lenz-Rezeption . . . . . . . . . . . 151

Lenz und die Wende zur "neuen Subjektivität" . . . . . . . 153

Lenz-Bezüge bei Hermann Kinder und Uwe Timm . . . . . . 158

Lenz-Bezüge bei Karin Struck . . . . . . . . . . . . . 160

Aneignungen der Lenz-Figur in Lyrik, Film, Oper

und Drama . . . . . . . . . . . . . . . . . . 162

Gert Hofmanns Lenz-Figur . . . . . . . . . . . . . . 167

Jochen Beyses Bezüge auf Lenz . . . . . . . . . . . . 169

Neueste Lenz-Rezeptionen in Oper, Film und Theater . . . . 171 
Werkverzeichnis und Sekundärliteratur ......... 175

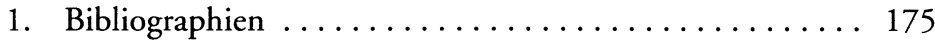

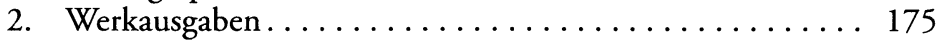

2.1 Werkausgaben zu Lenz' Lebzeiten ............. 175

2.2 Werkausgaben nach Lenz' Tod................ 175

2.3 Lenz' Werke in anderen Ausgaben . . . . . . . . . . 177

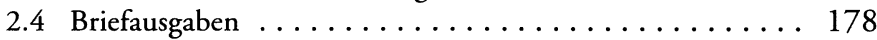

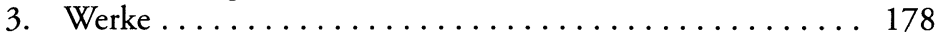

4. Sekundärliteratur zu Lenz................... 193

4.1 Übergreifende Sekundärliteratur . . . . . . . . . . . . 193

4.2 Sekundärliteratur zu einzelnen Werken ........... 206

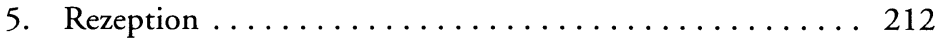

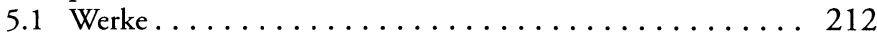

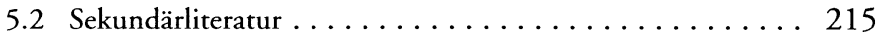

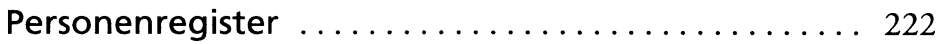

